\title{
Assessment of neutralizing antibody responses after natural SARS-CoV-2 infection and vaccination in Congolese individuals
}

Francine Ntoumi ( $\square$ fntoumi@fcrm-congo.com )

University of Tübingen

Armel Landry Batchi-Bouyou

Fondation Congolaise Pour La Recherche Médicale

Jean Claude Djontu

Fondation Congolaise Pour La Recherche Médicale

Jeannhey Christevy Vouvoungui

Fondation Congolaise Pour La Recherche Médicale

Claujens Chastel Mapanguy Mfoutou

Fondation Congolaise Pour La Recherche Médicale

Line Lobaloba Ingoba

Fondation Congolaise Pour La Recherche Médicale

Jiré Séphora Mougany

Fondation Congolaise Pour La Recherche Médicale

Kamal Rauchelvy Boumpoutou

Fondation Congolaise Pour La Recherche Médicale

Steve Diafouka-kietela

Fondation Congolaise Pour La Recherche Médicale

Raoul Ampa

Marien Ngouabi University

\section{Research Article}

Keywords: Vaccine, BBIP-CorV, Janssen/Ad26.COV2.S, SARS-CoV-2, antibodies, Republic of the Congo

Posted Date: February 23rd, 2022

DOI: https://doi.org/10.21203/rs.3.rs-1338684/v1

License: (c) (i) This work is licensed under a Creative Commons Attribution 4.0 International License.

Read Full License 


\section{Abstract}

\section{Background}

Assessing immune responses after vaccination is part of the evaluation package of vaccine effectiveness in the real world. With regard to SARS-CoV-2, neutralizing antibody levels has been shown to be a good indicator of antibody immune response boosting. So far, limited data have been reported from Africa including in Central Africa. The objective of this study was to provide data on anti-S1 spike total IgG and neutralizing antibodies in vaccinated and non-vaccinated including naturally infected Congolese population during B.1.214.1 and B.1.617.2 variant waves.

\section{Methods}

Recruited patients were divided into 4 groups: (1) Naturally infected by the B.1.214.1 variant on January 2021 and followed up until September 2021. These patients have been vaccinated at month 07 and then followed up for 2 months post vaccination; (2) Naturally infected by the B.1.617.2 variant from June 2021 ; (3) unvaccinated SARS-CoV-2 individuals with no history of prior SARS-CoV-2 infection; (3) fully vaccinated individuals with Sinopharm/BBIP-CorV or Janssen/Ad26.COV2.S. SARS-CoV-2 was detected by qRT-PCR and sequenced using Next-Generation Sequencing. ELISA method was used for detecting IgG, and neutralizing Antibody against SARS-CoV-2 antigens using commercial neutralizing assay.

\section{Results}

Individuals infected by the B.1214.1 variant elicited consistently high IgG titers at 02,03 and 06 months. Two months post vaccination with BBIP-CorV, participants showed a significant increase by $\times 2.5$ fold $(p<0.0001)$ of total IgG and X1.5 fold for neutralizing antibody capacity. This study showed that natural infection with B1.617.2 (delta) variant was more immunogenic compared to those being infected with B1.214.2 variant at the same period.

We found a significantly higher concentration in anti-SARS-CoV-2 IgG $(\mathrm{p}<0.0002)$ and antibodies neutralization capacity $(\mathrm{P}<0.0001)$ in fully vaccinated compared to unvaccinated participants. Two months post vaccination, individuals who received Janssen/Ad26.COV2.S presented higher $(p=0.01)$ total IgG to spike protein compared to BBIP-CorV.

\section{Conclusion}

Both natural infection and vaccination with BBIP-CorV and Janssen/Ad26.COV2.S induced antibody response in Congolese population. In addition, Janssen/Ad26.COV2.S was more immunogenic than Sinopharm/BBIP-CorV. There is a need to investigate the duration of these antibodies both in previously infected and naive vaccinated Congolese to allow public heath stakeholders to make evidence-based decision on vaccine schedule for the Congolese population.

\section{Background}


The new human viral pathogen, severe acute respiratory syndrome coronavirus-2 (SARS-CoV-2), the causative agent of the coronavirus disease 2019 (COVID-19) pandemic, emerged in China in December 2019. Since then, vaccines against COVID-19 developed at an unprecedented speed are currently rolled out all over the world (1). So far, even though hundreds of candidate vaccines have been developed worldwide (2), very limited data are reported about vaccine effectiveness in the African population which has reported the lowest number of cases and deaths/Million inhabitants since the beginning of the pandemic (3).

In Republic of the Congo, eligible persons for vaccination against COVID-19 should be 18 years-old or older including those who have been previously infected with SARS-CoV-2. As of December 31, 2021, approximately $10 \%$ of people who have been vaccinated in Republic of the Congo were fully vaccinated to an adenovirus vector vaccine (Janssen/Ad26.COV2.S) or an inactivated-virus vaccine BBIBPCorV/Sinopharm [SITREP 208]. With regard to the low vaccine coverage, high proportion of the population is exposed to natural infection particularly in Brazzaville and Pointe-Noire (4) which are the two main cities responsible for more than $90 \%$ of reported cases (SITREP 161).

Vaccines against SARS-CoV-2 have been shown to elicit levels of neutralizing antibodies comparable to those observed in naturally infected persons $(5,6)$. The presence of neutralizing antibodies from prior infection was significantly associated with protection against reinfection (7). However, it is still unclear which is the necessary titer of neutralizing antibodies that correlate with protection and how long neutralization activity persists in individuals in different conditions and geographical zones.

In 2021, the Republic of the Congo has faced 3 major COVID-19 waves caused by the B.1.214.1 (8) and B.1.617.2 (Delta) variants and vaccines were introduced in the country in March 2021. This has been an opportunity to enroll and follow up individuals naturally infected and those who received one or two complete doses of COVID-19 vaccine according to the vaccine injection scheme. The available baseline data regarding seroprevalence investigation in RoC dated from March to July 2020 in Brazzaville, the capital (4) and from July 2021 in the North of the country (9). The reported level of seroprevalence was about $27 \%$ in 2020 and between $25 \%$ and $67 \%$ in 2021. In some African countries, the seroprevalence is growing and expected to reach herd immunity $(10,11)$. Thus, reinfection cases $(12)$ show that it is not wise to rely on immunity acquired by natural infection to confer herd immunity;

The main objective of this study was to provide preliminary data on antibody immune responses to SARS-CoV-2 spike protein including total IgG and neutralizing capacity, in well characterized vaccinated and non-vaccinated including naturally infected Congolese population during B.1.214.1 and B1.617.2 variant waves that occurred in the country.

\section{Methods}

\section{Study design and population}


Patients were recruited at the health center of the Congolese Foundation for Medical Research in Massissia, southern district of Brazzaville from January 2021 to November 2021. The criteria for inclusion in the study were 1 ) to be aged 18 or older; 2 ) to provide informed consent to participate in the study; 3 ) to present clinical symptoms suggesting an infection with SARS-CoV-2. For all study participants, socio-demographic data were collected and combined with clinical data of symptoms (headache, fever, etc.). The vaccination status was recorded (the name of the vaccine and dates of vaccination).

Oropharyngeal swabs were collected from each participant for SARS-CoV-2 RT-PCR testing and blood sample for immune responses evaluation.

In the Republic of Congo, vaccination effort started on 24th March 2021. The vaccine coverage was about $1-5 \%$ during the study period [SITREP 137, SITREP 161].

The following case definitions were applied to enrolled patients:

1. Naturally infected by the B.1.214.1 variant on January 2021 and followed up until September 2021. These patients have been vaccinated at month 07 and then followed up 2 months post vaccination. Blood and oropharyngeal samples have been collected at 02, 03,06 and 08 months time points after enrolment.

2. Naturally infected by the B.1.617.2 variant from June 2021 (the first case of B.1.617.2 infected individual was mid June 2021 in RoC);

3. Unvaccinated SARS-CoV-2 individuals with no history of prior SARS-CoV-2 infection. As the vaccine hesitancy was high during the study period, many enrolled patients were not vaccinated and based on recruitment criteria, only individuals tested RT-PCR negative at enrolment and who reported that they have never been RT-PCR or antigen positive test for SARS-CoV-2 were included in this group.

4. A vaccinated group was established and only 2 months post-vaccination participants were enrolled in this study including BBIP-CorV (Sinopharm) or Janssen/Ad26.COV2.S (Johnson \& Johnson) vaccinees. Those who were RT-PCR positive at inclusion were excluded from the study.

Sequencing data reported in the study period showed that circulating strains were B1.214.1 and Delta (B1.1.617.2) variant.

\section{SARS-CoV-2 detection}

RNA was extracted from swabs using the QIAamp Viral RNA Mini Kit (Qiagen, Hilden, Germany) according to instructions and subjected to RealStar® SARS-CoV-2 real-time PCR targeting the S gene of SARS-CoV-2 (Altona Diagnostics, Hamburg, Germany) by using a high-performance, high-throughput PCR platform (96 well plates) LightCycler ${ }^{\circledR} 480$ Instrument II (Roche diagnostics, Mannheim, Germany). Amplicons with a $\mathrm{Ct}<30$ were sequenced using Next-Generation Sequencing (NGS).

\section{SARS-CoV-2 NGS sequencing}


Oxford Nanopore sequencing Technology (ONT) was used. Libraries were prepared as described in Freed et al., (RAPID barcoding, 1200bp amplicon) (13). The libraries were quantified (Qubit DNA BR, Thermo Scientific), and sequenced on ONT. The FastQ files obtained from sequencing were analyzed using artic

network field bioinformatics (14) pipeline for ONT data. Sequences were deposited in GISAID (15) and the lineages of these genomes were annotated by Pangolin online tool (16).

\section{SARS-CoV-2 specific antibodies detection Measurement of plasma IgG Ab}

The IgG Ab against SARS-CoV-2 antigens were measured using GSD NovaLisa ${ }^{\circledR}$ SARSCoV-2 (COVID-19) quantitative IgG (NovaTec Immundiagnostica $\mathrm{GmbH}$ ) according to the manufacturer's protocol. Sample preparation included the dilution of plasma with the dilution buffer (1:101). For the assay $100 \mu \mathrm{L}$ of Negative Control, Positive Control, and each diluted plasma were added to the corresponding wells of micro-plate pre-coated with SARS-CoV-2 antigens, and incubated at $37^{\circ} \mathrm{C}$ for $30 \mathrm{~min}$. After washing, 100 $\mu \mathrm{L}$ of enzyme substrate, tetramethyl benzidine (TMB), were added to each well, and incubated for 30 minutes at $37^{\circ}$ in the dark. Finally, $50 \mu \mathrm{L}$ of stop solution was added to each well and the optical density (OD) for each well immediately measured at $450 \mathrm{~nm}$ using an ELISA microplate reader. Quantitative results obtained in Arbitary Unit/ml (AU/ml) were converted to International Units (IU/ml) by multiplying 4.5 in accordance with WHO specifications. If the ratio was above was above $49.5 \mathrm{IU} / \mathrm{ml}$, it was considered as positive. Negative Control, Positive Control, and Calibrator Control (mix of Positive Control with Negative Control) were included in each assay for quality control.

\section{Measurement of anti-SARS-CoV-2 neutralizing antibodies}

The commercialized cPass ${ }^{\text {TM }}$ SARS-CoV-2 Neutralization Antibody Detection Kit (Nanjing GenScript Biotech, China) including the peroxydase conjugated Spike protein receptor binding domain (HRP-RBD) was used for the detection of neutralizing anti-SARS-CoV-2 Antibodies according to manufacturer's protocol and published reports $(5,7,9,24,32)$. Both negative and positive controls were included in each assay for quality control. A sample was declared positive for neutralizing antibodies if its inhibition was at least $30 \%$.

\section{Statistical analysis}

The data were analyzed using SPSS version 24 (SPSS Inc., Chicago, IL, USA). GraphPad (version 8.0.4) was used to generate the figures. Categorical variables were presented as numbers (\%). Continuous variables were expressed as median (interquartile range, IQR) or mean ( \pm standard deviation, SD). MannWhitney U-test or Kruskal-Wallis test were used for observing significant difference in distributions between two or more groups. For continuous variables $\mathrm{x} 2$ or Fisher's exact test were used. Statistical significance was defined as $P$ values of $<0.05$.

\section{Results}




\section{Follow up of IgG and neutralizing antibodies to spike protein after natural infection and subsequent vaccination with BBIP-CorV}

A total of 17 participants were enrolled in January 2021 according to inclusion criteria and followed up during 8 months. The average age was $43.9 \pm 9.8$ (31 to 66 years old) including 13 males and 4 females for sex ratio of 3.3 .

The levels of total IgG to the spike protein remain stable and high corresponding to X2-3 fold the threshold $=50 \mathrm{IU} / \mathrm{mL}$, at 02, 03 and 06 months post natural infection with B.1.214.1 variant. After two months post vaccination with BBIP-CorV vaccine, a significant increase by $\times 2.5$ fold was observed $(p<$ 0.001). (Fig. 1A).

With regard to the neutralizing antibodies, 2, 3 and 6 months post natural infection the neutralizing capacity is high and remain also stable over 6 months and the vaccination significantly increase by $x 1.5$ fold the neutralizing capacity of antibodies (Fig. 1B).

\section{Assessment of antibody response against natural infection with B1.214.1 and B1.617.2 (Delta) variant in Congolese patients}

A total of 69 SARS-CoV-2 RT-PCR positive individuals were enrolled. The infecting SARS-CoV-2 strains was identified and a blood sample collected two months after acute infection. We recruited 17 and 52 individuals infected with B1.214.1 and Delta variant respectively.

As shown in Fig. 2A, IgG antibodies is significantly $(p=0.011)$ lower in individuals infected by B1.214.1 compared to those infected with Delta variant two months post-infection. Moreover, a trend to higher \% inhibition of neutralizing antibodies was observed in patients who were infected with Delta variant $(\mathrm{P}=$ 0.083) (Fig. 2B). The difference in IgG antibody levels was $x 3$ fold lower and the inhibition of neutralizing capacity was X 2.5 lower in patients who have been infected by B1.241.1 (respectively Fig. 2A and 2B). Therefore, infection with Delta variant is stronger stimulator of antibody responses and neutralizing capacity.

\section{IgG antibody responses and neutralizing capacity in vaccinated and non-vaccinated Congolese individuals}

A total of fifty vaccinees were enrolled two months post vaccination. They were matched with 50 individuals non vaccinated (group 2, Materials and methods). The demographic characteristics of enrolled vaccinees are presented in Table 1. Twenty-four and twenty-six participants received BBIP-CorV (Sinopharm) and Janssen/Ad26.COV2.S (Johnson \& Johnson) vaccine respectively. Figure 3A and B 
show a significant difference $(p<0.0002)$ in IgG to spike and \% inhibition of neutralization $(P<0.0001)$ between vaccinated and unvaccinated participants.

Table 1

Demographic characteristics of Congolese participants immunized with SARS-CoV-2 vaccines BBIP-CorV or Ad26.COV2.S

\begin{tabular}{|c|c|c|c|c|}
\hline \multirow[t]{2}{*}{ Variables } & \multicolumn{3}{|l|}{ Vaccinated } & \multirow[t]{2}{*}{ Unvaccinated } \\
\hline & $\begin{array}{l}\text { BBIP-CorV } \\
\mathrm{N}=24\end{array}$ & $\begin{array}{l}\text { Janssen/Ad26.COV2.S } \\
N=26\end{array}$ & $\begin{array}{l}\mathrm{P} \text { - } \\
\text { value }\end{array}$ & \\
\hline Age (years) (IQR) & $\begin{array}{l}45(34.25 \\
56)\end{array}$ & $34.50(26 ; 49.75)$ & & $\begin{array}{l}38(27.75 \\
47)\end{array}$ \\
\hline Gender n(\%) & $10(41.7)$ & 14(63.9) & 0.412 & $22(44)$ \\
\hline Female & $14(58.3)$ & $12(46.1)$ & & $28(56)$ \\
\hline \multicolumn{5}{|l|}{ Male } \\
\hline IgG results n(\%) & $5(20.8)$ & $3(11.6)$ & 0.456 & $29(58)$ \\
\hline Negative & 19(79.2) & $23(88.4)$ & & $21(42)$ \\
\hline \multicolumn{5}{|l|}{ Positive } \\
\hline \multirow{2}{*}{$\begin{array}{l}\text { Median IgG concentration } \\
(\mathrm{Ul} / \mathrm{ml})(\mathrm{IQR})\end{array}$} & 115 & 550.9 & 0.014 & 58.81 \\
\hline & $\begin{array}{l}(50.14 ; \\
318.1)\end{array}$ & $(74.31 ; 1085)$ & & $\begin{array}{l}(38.07 \\
160.1)\end{array}$ \\
\hline \multirow{2}{*}{$\begin{array}{l}\text { Neutralizing antibody results } \\
\mathrm{n}(\%)\end{array}$} & $7(29.2)$ & $6(23)$ & 0.750 & $23(46)$ \\
\hline & 17(70.8) & $20(77)$ & 0.431 & $27(54)$ \\
\hline Negative & 62.23 & 87.81 & 0.912 & 30.75 \\
\hline \multirow{2}{*}{$\begin{array}{l}\text { Positive } \\
\text { Median \%inhibition (IQR) }\end{array}$} & (14.01; & $(30.65 ; 91.14)$ & & $(4.61 ; 73.82)$ \\
\hline & प्र1.51) & & & \\
\hline
\end{tabular}

The proportion of people with neutralizing antibodies is significantly higher in vaccinated participants in $80 \%$ of cases $(n=40 / 50)$ compared to unvaccinated participants in $54 \%$ of cases $(n=27 / 50)$ who also reported to have never been infected by SARS-CoV-2 ( $p=0.004)$. Even so, more than $50 \%$ of unvaccinated participants had neutralizing antibodies against SARS-CoV-2 spike protein (Fig. 3C and D). Identically, $90 \%(n=45 / 50)$ of vaccinees harbored IgG versus $58 \%(n=29 / 50)$ in the unvaccinated group $(p=$ $0.0002)$.

\section{Comparison between vaccine-induced antibody response from BBIP-CorV and Janssen/Ad26.COV2.S}


The two groups of individuals who received BBIP-CorV and Janssen/Ad26.COV2.S vaccines were quite homogeneous in term of gender and age. The number of participants with IgG above the threshold is similar (79\% vs $88 \%$ for BBIP-CorV versus Janssen/Ad26.COV2.S) in both groups of vaccinees. A significant difference $(p<0.014)$ in the median concentration of IgG observed.

Two months post vaccination showed a significant difference in total IgG $(P=0.002)$ (Fig. 4B) in Janssen/Ad26.COV2.S group compared to BBIP-CorV vaccine group. Similarly, neutralization antibody response was slightly higher in Janssen/Ad26.COV2.S group compared to BBIP-CorV vaccine $(p=0.06)$.

The number of vaccinated participants harboring IgG to spike protein was not significantly higher in Janssen/Ad26.COV2.S group (94\%) vs $82 \%$ for BBIP-CorV vaccine group. The number of vaccinees with antibodies capacity to neutralize SARS-CoV-2 was also not significantly higher for Janssen/Ad26.COV2.S group (83\% vs $74 \%$ ) (Fig. 4).

\section{Discussion}

Vaccination has a pivotal role for infectious diseases pandemic eradication. Although, COVID-19 cases continue to increase as variants surge, we are experiencing in Sub-Saharan Africa a stagnation of vaccination acceptance rate (17). In the Republic of the Congo, the vaccination coverage is as low as $10 \%$ after one year of vaccine rollout [SITREP 215, 25 January 2022]. Despite the laudable efforts of governments to acquire vaccines through donation or purchase for a targeting coverage of $60 \%$ of the population, the key question that is being asked not only by the local stakeholders but also by the population is how effective they are in the African population considering the different genetic background as well as the environmental context. The negative attitudes and perceptions regarding COVID-19 vaccines in Central Africa might be attributed to limited evidence of their effectiveness, making it imperative to conduct the current study.

Congolese individuals naturally infected by with B.1214.1 SARS-CoV-2 variant harbored high and stable titers of IgG and neutralizing capacity over 6 months, which were boosted significantly by vaccination. Previous published studies conducted in Asia, Europe and USA indicated that S-specific antibodies dynamic remained relatively stable post-infection and started waning at six months (18-21). In contrast to these studies which generally included participants regardless of the SARS-CoV-2 infecting variant, our study focused specifically on antibodies kinetic after infection with the B.1.214.1 variant. The B.1.214.1 variant linked to the parent lineage B.1.214 $(8,22)$ was predominant during the early phases of the pandemic in Republic of the Congo and possibly in other neighboring countries with limited genomic surveillance (23). The longitudinal study showed that all patients infected by B.1.124.1 variant were tested negative at each checkpoint follow-up, suggesting that the SARS-CoV-2 antibody response induced would have protected them from reinfections by emerging variants.

Consistent with previous observations (24), the generation and maintenance of neutralizing antibodies against SARS-CoV-2 play an important role in resisting reinfections and could be an important indicator for protection $(7,25)$. This means that the combined effects of waning antibody responses and increased 
risk of reinfections suggest that vaccination might be needed to potentialize protection. Our analysis suggests that maximizing neutralizing antibody responses through booster vaccination of previously infected individuals, should be an effective strategy to broadly increase neutralization titers against SARS-CoV-2 variants (26). In addition, vaccination of previously infected individuals occurred around 6 months after infection. Most boosting studies in previously infected individuals suggested there is a benefit in delaying to 6 months (27). If fact, 6 months interval is an optimal timeframe enabling an increase in the number of memory B cells after infection responsible of better antibody responses after boosting (28).

Similar to many investigations that evaluated IgG antibody levels after vaccination (29-31), here the groups of naive participants have been followed up two months after vaccination and compared to matched naïve unvaccinated participants. Without any surprise, unvaccinated group showed significantly lower total IgG and neutralizing capacity compared to vaccinated (whatever the used vaccine). This result is important because it shows that in the general Congolese population exposed to SARS-CoV-2 virus and not tested, needs to be immunized through vaccination for efficient boosting their immunity to COVID-19. To date, this finding is the first report showing the importance of vaccination in the Congolese environment and this local evidence is very important to respond to vaccine hesitancy.

When comparing the antibody response from delta variant with that from B.1.214.1, higher levels IgG and neutralizing antibodies were observed with the Delta variant compared to the B.1.214.1 variant 2 months after infection. This may be explained by the fact that the delta variant contains mutations within the spike protein including in the sites specific to neutralizing antibodies, that might lead to the more immunogenic epitopes (32-34). Furthermore, the high viral load caused by the Delta variant which has been reported to be ten times higher than historical SARS-CoV-2 variants, resulting in the production of high levels of neutralizing antibodies needed to inhibit the RBD-ACE2 complex (35).

To our knowledge, this study is the first to report immunological data on BBIBP-CorV vaccine in the African population. As BBIBP-CorV vaccines largely deployed on the African continent, this information is crucial for national stakeholders as it is difficult to make decision based on the immense amount of data (36-38) from mRNA vaccine rolled out in Europe and United states.

Using the fact that Janssen/Ad26.COV2.S and BBIBP-CorV vaccines were rolled out in the republic of Congo, this study compared immunological responses due to both vaccines. It was found that Janssen/Ad26.COV2.S, adenovirus based vaccine(39) induced higher antibody response than BBIP-CorV. BBIBP-CorV which is an inactivated virus vaccine. Based on literature (40), inactivated virus vaccines have stable conformation-dependent epitope expression and are expected to better enhance the humoralbased response whereas adenovirus vector vaccines have the advantage to harbor a persistence of antigen expression that has been reported to contribute to prolonged immune responses and long-lasting immunity than inactivated virus vaccines (41). This preliminary result is very interesting and raise the issue of duration of these IgG and neutralizing antibodies in both vaccinated groups. It would be 
important to investigate further duration of these antibodies in a larger group of individuals. However, the limited $(N=50)$ number of participants have been addressed by using a test negative design study.

\section{Conclusions}

Both natural infection and vaccination by BBIP-CorV and Janssen/Ad26.COV2.S induced antibody response in Congolese population. Here we found that adenovirus-based vaccine was more able to induce higher total IgG and antibodies neutralizing capacity compared to inactivated virus vaccine. There is a need to investigate now the duration of these antibodies both in previously infected and naive vaccinated Congolese recipients to allow public heath stakeholders to decide on vaccine schedule in the Congolese population.

\section{Abbreviations}

ACE2: Angiotensin-converting enzyme 2

ELISA : Enzyme-Linked Immuno Assay

GISAID : Global Initiative on Sharing Avian Influenza Data

IgG: Immunoglobulin G

NGS: Next-Generation-Sequencing

ONT : Oxford Nanopore sequencing Technology

OD : Optic density

RBD: Receptor-binding domain

RT-PCR: Reverse Transcription Polymerase Chain Reaction

SARS-CoV-2: severe acute respiratory syndrome coronavirus 2

SITREP: Situation Report

WHO : World Health Organization

\section{Declarations}

\section{Ethics approval and consent to participate}

The study was approved by the Institutional Ethics Committee of the Congolese Foundation for Medical Research. Informed consent has been obtained by a qualified member of the investigation team, for all 
persons wishing to participate in the study, and before any start of any procedure planned within the framework of the investigation. Each participant was informed that his/her participation in the survey was voluntary and that they were free to withdraw, without justification, from the survey at any time. All methods in this study were performed in accordance with the relevant guidelines and regulations

\section{Consent for publication}

Not applicable

\section{Availability of data and materials}

The datasets generated and/or analyzed during the current study are not publicly available due to terms and conditions defined by the local Ethical committee, but are available from the corresponding author on reasonable request.

\section{Competing interests}

There are no competing interests for any of the authors of this manuscript.

\section{Funding}

This research was funded by Fondation Congolaise pour la Recherche Médicale, Humboldt Research Hub COvid-19 in Central Africa, ORCHESTRA (GRANT NUMBER 101016167), the World Health Organization, Bill and Melinda Gates Foundation, GISAID and PANDORA-ID-Net (EDCTP Grant Agreement RIA2016E1609). The funders had no role in design, analysis or decision to submit this manuscript.

\section{AUTHOR'S CONTRIBUTIONS}

Affiliations

Fondation Congolaise pour la Recherche Médicale (FCRM), Brazzaville, Republic of Congo

Armel Landry Batchi-Bouyou, Jean Claude Djontu, Jeannhey Christevy Vouvoungui, Claujens Chastel Mapanguy Mfoutou, Line Lobaloba Ingoba, Jiré Séphora Mougany, Kamal Rauchelvy Boumpoutou, Steve Diafouka-kietela, Francine Ntoumi

Faculty of Sciences and Techniques, University Marien Ngouabi, Brazzaville, Republic of Congo Armel Landry Batchi-Bouyou, Claujens Chastel Mapanguy Mfoutou, Line Lobaloba Ingoba, Jiré Séphora Mougany, Raoul Ampa 
Institute of Tropical Medicine, University of Tübingen, Tübingen, Germany

Francine Ntoumi

\section{Contributions}

ALBB, FN conceived the study. ALBB, FN wrote the first draft. ALBB, JCD, CCMM, LLI, JSM, KRB, SDK collected and preprocessed the data. ALBB, JCD, JCV, did the main analysis. ALBB, FN, JCD, RA wrote the main text. All authors reviewed and approved the final manuscript.

\section{ACKNOWLEDGEMENTS}

We thank all the volunteers who accepted to participate in this study

\section{References}

1. Alkandari D, Herbert JA, Alkhalaf MA, Yates C, Panagiotou S. SARS-CoV-2 vaccines: fast track versus efficacy. The Lancet Microbe. 2021;2(3):e89-e90.

2. WHO. Draft landscape and tracker of COVID-19 candidate vaccines. Accessed January 23, 2021 [Available from: https://www.who.int/publications/m/item/draft-landscape-of-covid-19-candidatevaccines.

3. CDC A. [Available from: https://africacdc.org/covid-19/.

4. Batchi-Bouyou AL, Lobaloba Ingoba L, Ndounga M, Vouvoungui JC, Mfoutou Mapanguy CC, Boumpoutou KR, et al. High SARS-CoV-2 IgG/IGM seroprevalence in asymptomatic Congolese in Brazzaville, the Republic of Congo. International journal of infectious diseases: IJID : official publication of the International Society for Infectious Diseases. 2021;106:3-7.

5. Ju B, Zhang Q, Ge J, Wang R, Sun J, Ge X, et al. Human neutralizing antibodies elicited by SARS-CoV2 infection. Nature. 2020;584(7819):115-9.

6. Liu L, Wang P, Nair MS, Yu J, Rapp M, Wang Q, et al. Potent neutralizing antibodies against multiple epitopes on SARS-CoV-2 spike. Nature. 2020;584(7821):450-6.

7. Addetia A, Crawford KHD, Dingens A, Zhu H, Roychoudhury P, Huang ML, et al. Neutralizing Antibodies Correlate with Protection from SARS-CoV-2 in Humans during a Fishery Vessel Outbreak with a High Attack Rate. Journal of clinical microbiology. 2020;58(11).

8. Ntoumi F, Mfoutou Mapanguy CC, Tomazatos A, Pallerla SR, Linh LTK, Casadei N, et al. Genomic surveillance of SARS-CoV-2 in the Republic of Congo. International Journal of Infectious Diseases. 2021;105:735-8.

9. Lobaloba Ingoba L, Djontu JC, Mfoutou Mapanguy CC, Mouzinga F, Diafouka Kietela S, Vouvoungui $\mathrm{C}$, et al. Seroprevalence of anti-SARS-CoV-2 antibodies in a population living in Bomassa village, Republic of Congo. International Journal of Infectious Diseases Regions.2022 Vol2 March: 130-136. 
10. Mukwege D, Byabene AK, Akonkwa EM, Dahma H, Dauby N, Cikwanine Buhendwa J-P, et al. High SARS-CoV-2 Seroprevalence in Healthcare Workers in Bukavu, Eastern Democratic Republic of Congo. Am J Trop Med Hyg. 2021;104(4):1526-30.

11. Ngere I, Dawa J, Hunsperger E, Otieno N, Masika M, Amoth P, et al. High Seroprevalence of SARSCoV-2 Eight Months After Introduction in Nairobi, Kenya. SSRN; 2021.

12. Iwasaki A. What reinfections mean for COVID-19. The Lancet Infectious Diseases. 2021;21(1):3-5.

13. Freed NE, Vlkova M, Faisal MB, Silander OK. Rapid and inexpensive whole-genome sequencing of SARS-CoV-2 using 1200 bp tiled amplicons and Oxford Nanopore Rapid Barcoding. Biol Methods Protoc. 2020;5(1):bpaa014.

14. Github. [Available from: https://github.com/artic-network/fieldbioinformatics.

15. Shu Y, McCauley J. GISAID: Global initiative on sharing all influenza data - from vision to reality. Euro Surveill. 2017;22(13).

16. O'Toole A, Scher E, Underwood A, Jackson B, Hill V, McCrone JT, et al. Assignment of epidemiological lineages in an emerging pandemic using the pangolin tool. Virus Evol. 2021;7(2):veab064.

17. Mutombo PN, Fallah MP, Munodawafa D, Kabel A, Houeto D, Goronga T, et al. COVID-19 vaccine hesitancy in Africa: a call to action. The Lancet Global health. 2021.

18. Dan JM, Mateus J, Kato Y, Hastie KM, Yu ED, Faliti CE, et al. Immunological memory to SARS-CoV-2 assessed for up to 8 months after infection. Science. 2021;371(6529).

19. Chia WN, Zhu F, Ong SWX, Young BE, Fong S-W, Le Bert N, et al. Dynamics of SARS-CoV-2 neutralising antibody responses and duration of immunity: a longitudinal study. The Lancet Microbe. 2021;2(6):e240-e9.

20. García-Abellán J, Padilla S, Fernández-González M, García JA, Agulló V, Andreo M, et al. Antibody Response to SARS-CoV-2 is Associated with Long-term Clinical Outcome in Patients with COVID-19: a Longitudinal Study. Journal of Clinical Immunology. 2021;41(7):1490-501.

21. Shioda K, Lau MS, Kraay AN, Nelson KN, Siegler AJ, Sullivan PS, et al. Estimating the cumulative incidence of SARS-CoV-2 infection and the infection fatality ratio in light of waning antibodies. Epidemiology (Cambridge, Mass). 2021;32(4):518.

22. Gerdol M. Emergence of a recurrent insertion in the N-terminal domain of the SARS-CoV-2 spike glycoprotein. bioRxiv. 2021:2021.04.17.440288.

23. Brito AF, Semenova E, Dudas G, Hassler GW, Kalinich CC, Kraemer MU, et al. Global disparities in SARS-CoV-2 genomic surveillance. medRxiv. 2021.

24. Jiang S, Hillyer C, Du L. Neutralizing antibodies against SARS-CoV-2 and other human coronaviruses. Trends in immunology. 2020;41(5):355-9.

25. Huang AT, Garcia-Carreras B, Hitchings MD, Yang B, Katzelnick LC, Rattigan SM, et al. A systematic review of antibody mediated immunity to coronaviruses: kinetics, correlates of protection, and association with severity. Nature communications. 2020;11(1):1-16. 
26. Cavanaugh AM, Spicer KB, Thoroughman D, Glick C, Winter K. Reduced risk of reinfection with SARSCoV-2 after COVID-19 vaccination-Kentucky, May-June 2021. Morbidity and Mortality Weekly Report. 2021;70(32):1081.

27. Pan H, Wu Q, Zeng G, Yang J, Jiang D, Deng X, et al. Immunogenicity and safety of a third dose, and immune persistence of CoronaVac vaccine in healthy adults aged 18-59 years: interim results from a double-blind, randomized, placebo-controlled phase 2 clinical trial. MedRxiv. 2021.

28. Wheatley AK, Juno JA, Wang JJ, Selva KJ, Reynaldi A, Tan H-X, et al. Evolution of immune responses to SARS-CoV-2 in mild-moderate COVID-19. Nature communications. 2021;12(1):1-11.

29. Krammer F, Srivastava K, Alshammary H, Amoako AA, Awawda MH, Beach KF, et al. Antibody responses in seropositive persons after a single dose of SARS-CoV-2 mRNA vaccine. New England Journal of Medicine. 2021;384(14):1372-4.

30. Prendecki M, Clarke C, Brown J, Cox A, Gleeson S, Guckian M, et al. Effect of previous SARS-CoV-2 infection on humoral and T-cell responses to single-dose BNT162b2 vaccine. The Lancet. 2021;397(10280):1178-81.

31. Manisty C, Otter AD, Treibel TA, McKnight Á, Altmann DM, Brooks T, et al. Antibody response to first BNT162b2 dose in previously SARS-CoV-2-infected individuals. The Lancet. 2021;397(10279):10578.

32. Edara VV, Norwood C, Floyd K, Lai L, Davis-Gardner ME, Hudson WH, et al. Infection-and vaccineinduced antibody binding and neutralization of the B. 1.351 SARS-CoV-2 variant. Cell Host \& Microbe. 2021;29(4):516-21. e3.

33. Liu Z, VanBlargan LA, Bloyet L-M, Rothlauf PW, Chen RE, Stumpf S, et al. Identification of SARS-CoV2 spike mutations that attenuate monoclonal and serum antibody neutralization. Cell host \& microbe. 2021;29(3):477-88. e4.

34. Plante JA, Mitchell BM, Plante KS, Debbink K, Weaver SC, Menachery VD. The variant Gambit: COVID's next move. Cell host \& microbe. 2021.

35. Teyssou E, Delagrèverie H, Visseaux B, Lambert-Niclot S, Brichler S, Ferre V, et al. The Delta SARSCoV-2 variant has a higher viral load than the Beta and the historical variants in nasopharyngeal samples from newly diagnosed COVID-19 patients. J Infect. 2021;83(4):e1-e3.

36. Tenforde MW, Patel MM, Ginde AA, Douin DJ, Talbot HK, Casey JD, et al. Effectiveness of severe acute respiratory syndrome coronavirus 2 messenger RNA vaccines for preventing coronavirus disease 2019 hospitalizations in the United States. Clinical Infectious Diseases. 2021.

37. Pouwels KB, Pritchard E, Matthews PC, Stoesser N, Eyre DW, Vihta K-D, et al. Effect of Delta variant on viral burden and vaccine effectiveness against new SARS-CoV-2 infections in the UK. Nature medicine. 2021;27(12):2127-35.

38. Paris C, Perrin S, Hamonic S, Bourget B, Roué C, Brassard O, et al. Effectiveness of mRNA-BNT162b2, mRNA-1273, and ChAdOx1 nCoV-19 vaccines against COVID-19 in healthcare workers: an observational study using surveillance data. Clinical Microbiology and Infection. 2021;27(11):1699. e5-. e8. 
39. Trovato M, Sartorius R, D’Apice L, Manco R, De Berardinis P. Viral emerging diseases: challenges in developing vaccination strategies. Frontiers in Immunology. 2020;11.

40. Dong Y, Dai T, Wei Y, Zhang L, Zheng M, Zhou F. A systematic review of SARS-CoV-2 vaccine candidates. Signal transduction and targeted therapy. 2020;5(1):1-14.

41. Coughlan L. Factors which contribute to the immunogenicity of non-replicating adenoviral vectored vaccines. Frontiers in immunology. 2020;11:909.

\section{Figures}

\section{Figure 1}

anti-SARS-CoV-2 antibody response to the B.1.214.1 variant two, three and six months after recovery, and 2 months after vaccination. Graph A shows the dynamic of IgG concentration. The percentage of inhibition of neutralizing antibodies before and after receiving vaccine is shown in graph B. KruskalWallis-test was used for the comparison. 


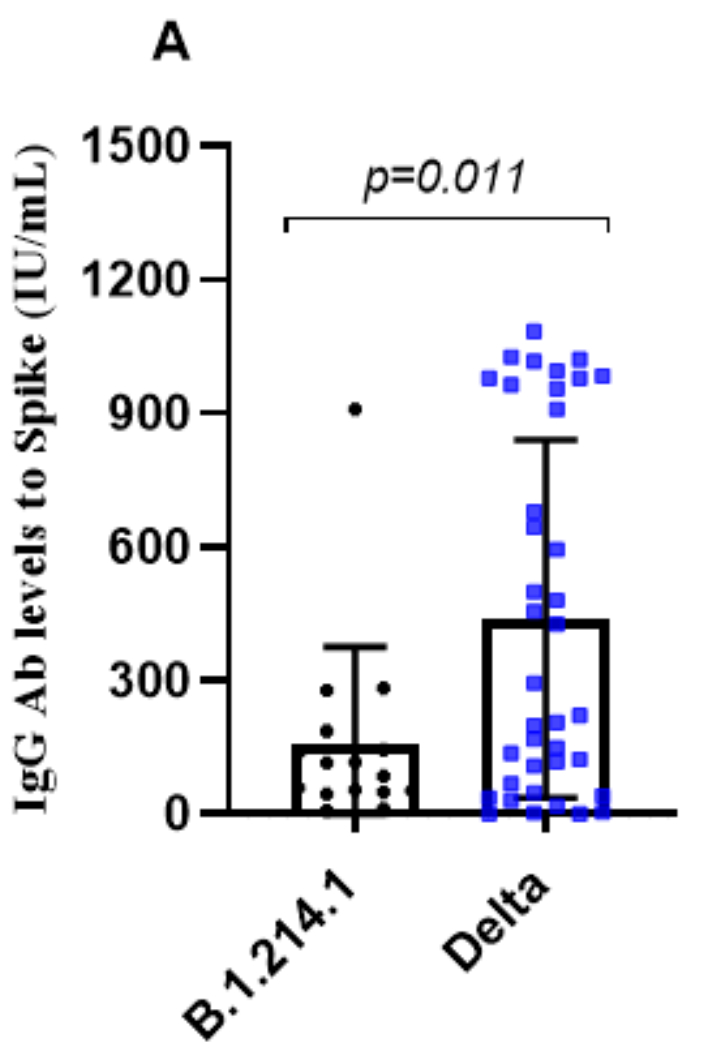

SARS-CoV-2 variants

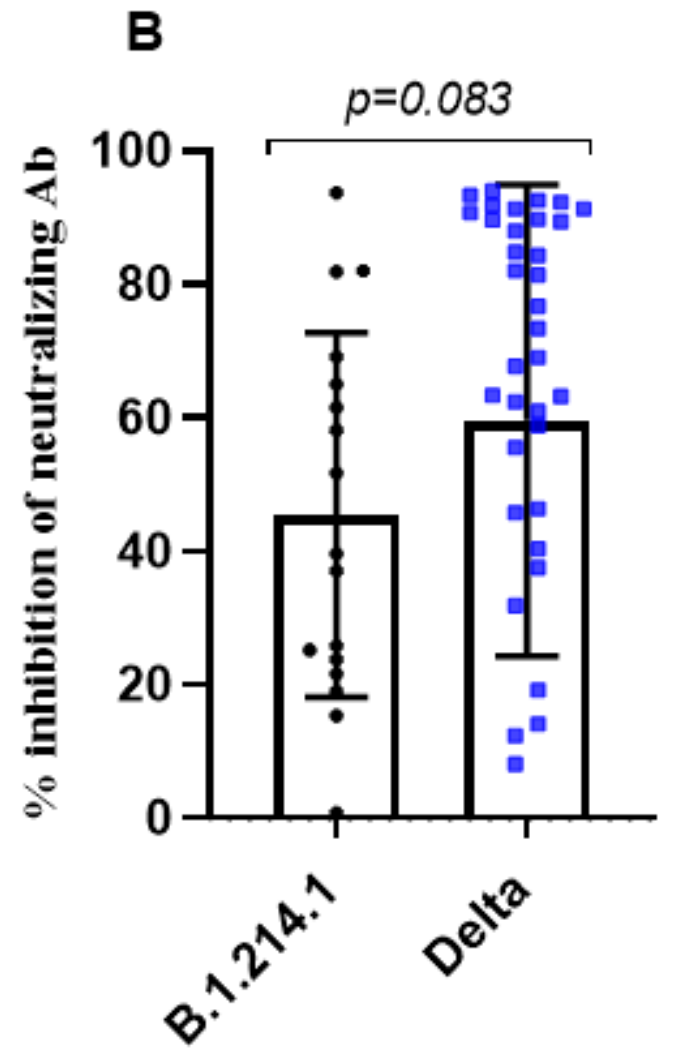

SARS-CoV-2 variants

Figure 2

anti-SARS-CoV-2 antibody response to the both B.1.214.1 and Delta variants 2 months after infection screening. The concentration of the IgG level is found in Graph A. The Inhibition percentage of neutralization between B.1.214.1 and Delta variant is shown in Graph B. Mann-Whitney Rank Sum test was used for the comparison between two groups.

Figure 3

Comparison of antibody responses between vaccinated and unvaccinated. Graph A shows the percentage of inhibition of neutralizing antibodies. The concentration of IgG is represented in graph $\mathbf{B}$. Graph $\mathbf{C}$ shows the proportions of people having neutralizing antibodies and graph $\mathbf{D}$ represents the proportions of participants having IgG antibodies. Mann-Whitney Rank Sum test was used for the comparison of antibodies levels and neutralization capacity, and Fisher exact test for the comparison of percentage of participants with IgG and neutralizing antibodies between two groups. 


\section{Figure 4}

Comparison of antibody responses between Sinopharm BBIBP-CorV vaccine and Janssen/Ad26.COV2.S. Graph A shows the percentage of inhibition of neutralizing antibodies. The concentration of IgG is represented in graph B. Graph $\mathbf{C}$ shows the proportions of people having neutralizing antibodies and graph D represents the proportions of participants having IgG antibodies. Mann-Whitney Rank Sum test was used for the comparison of antibodies levels and neutralization capacity, and Fisher exact test for the comparison of percentage participants with IgG and neutralizing antibodies between two groups. 